\title{
Paludisme
}

\section{et grossesse : \\ un dilemme \\ thérapeutique}

François Nosten

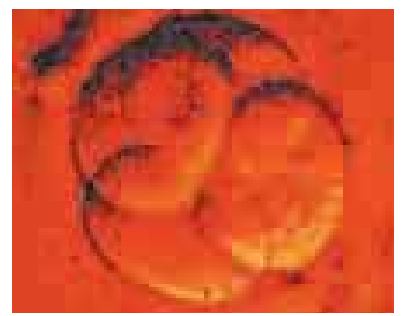

Shoklo Malaria Research Unit, SMRU, PO Box 46, Mae Sot 63110, Thaillande.

francois@tropmedres.ac www.shoklo-unit.com
Le paludisme est la parasitose humaine la plus meurtrière. Chaque année, le nombre des malades atteint des centaines de millions et le nombre de décès plusieurs millions. La moitié de la population du globe y est exposée. Son poids sur les économies les plus faibles est considérable et, depuis une dizaine d'années, des efforts renouvelés ont été entrepris pour lutter contre ce fléau. De fait, le paludisme n'est plus à proprement parler une maladie négligée. Sauf pour ce qui concerne la femme enceinte.

\section{Épidémiologie du paludisme de la femme enceinte}

On sait depuis les années 1950 que la grossesse (en particulier la première) rend la femme plus susceptible à l'infection palustre. En zone de forte transmission, la mère souffre d'anémie parfois sévère, voire mortelle, le fœtus d'un retard de croissance in utero et le nouveau-né d'un faible poids à la naissance. Dans les zones de transmission faible ou instable (et en particulier lors d'épidémies), la femme enceinte peut succomber à un paludisme pernicieux et les accès de fièvres sont responsables d'avortements, d'enfants mort-nés et de prématurité [1]. Malgré les études épidémiologiques nombreuses en Afrique et ailleurs, les statistiques fiables manquent sur la mortalité maternelle attribuée au paludisme. On estime cependant que chaque année des centaines de milliers de femmes meurent pendant la grossesse des effets directs (paludisme sévère) ou indirects (anémie) de l'infection palustre. À cette mortalité maternelle, il faut ajouter celle des nouveau-nés, prématurés ou de faible poids, et les grossesses interrompues. En Afrique sub-saharienne, le paludisme est presque exclusivement dû à Plasmodium falciparum, mais dans le reste du monde (beaucoup plus peuplé) le Plasmodium vivax coexiste avec $P$. falciparum. Les effets de l'infection à $P$. vivax pendant la grossesse, récemment décrits, sont moins sévères mais associent anémie maternelle et réduction du poids de naissance [2]. Comme pour l'infection à $P$. falciparum, les accès fébriles dus à $P$. vivax sont aussi responsables d'accouchements prématurés et d'interruptions de grossesses. Le paludisme congénital est peu fréquent mais pas exceptionnel. Enfin la co-infection avec le VIH (virus de l'immunodéficience humaine) aggrave le paludisme de la femme enceinte et favorise l'infestation placentaire et le passage transplacentaire des parasites.

\section{Briser le cercle vicieux de l'absence d'antipaludiques efficaces utilisables chez la femme enceinte}

Toutes ces données épidémiologiques sur les effets désastreux du paludisme pendant la grossesse pourraient faire penser que nous disposons aujourd'hui de stratégies robustes pour le traitement et la prévention du paludisme maternel. II n'en est rien. La plupart des programmes nationaux de lutte contre le paludisme n'ont aucune recommandation spécifique pour le traitement du paludisme gestationnel. Même dans les pays les plus développés, les traitements utilisés pour traiter les cas de paludisme d'importation sont souvent inadaptés à l'évolution de la chimiorésistance. 
Cette dernière, en grande partie responsable de la résurgence du paludisme à l'échelle planétaire (et donc du paludisme d'importation), compromet aussi les stratégies de chimioprévention (prophylaxie ou traitement intermittent) et a mis à jour d'importantes lacunes dans nos connaissances sur les antipaludiques et leur utilisation pendant la grossesse. Depuis le drame de la thalidomide, très peu de médicaments ont été développés pour une indication chez la femme enceinte. Parmi le petit nombre d'antipaludiques mis sur le marché dans les vingt dernières années (faute de marché l'industrie pharmaceutique investit très peu dans ces médicaments), tous excluent la grossesse (et parfois l'allaitement) dans leurs indications. Par précaution d'ailleurs plutôt que par l'existence de données avérées suggérant une toxicité embryonnaire. Ces produits ne sont donc pas utilisés chez les femmes enceintes et les données restent inexistantes. Voici donc le cercle vicieux qu'il faut briser pour que les femmes enceintes atteintes de paludisme puissent bénéficier d'autres traitements que ceux qui sont devenus inefficaces.

\section{Pourquoi les schémas d'utilisation des antipaludiques chez la femme enceinte sont-ils inadéquats ?}

Parmi les médicaments dont nous disposons, seules la primaquine et les tétracyclines sont clairement contre-indiquées chez la femme enceinte. La chloroquine, l'amodiaquine et la sulfadoxine-pyriméthamine sont considérées comme sûres mais elles sont malheureusement souvent inefficaces. La quinine aussi peut être prescrite mais elle est très mal tolérée et provoque des hypoglycémies. Par ailleurs son efficacité a aussi beaucoup diminué, en particulier en Asie [3]. Les femmes enceintes étant systématiquement exclues des essais cliniques, aucune donnée n'existe sur les risques associés à l'administration pendant la grossesse de méfloquine, des dérivés de l'artémisinine, de l'atovaquone-proguanil, de la pipéraquine dans les dossiers cliniques du développement de ces médicaments. Dans ces conditions, comment traiter une femme enceinte atteinte de paludisme multirésistant? De façon paradoxale on oppose souvent des arguments relevant de l'éthique au recrutement de femmes enceintes dans les études de thérapeutique. Le risque supposé ou réel pour le fœtus étant considéré comme supérieur au bénéfice pour la mère, pourtant atteinte d'une infection aux conséquences redoutables. II n'est pas rare encore aujourd'hui de constater que des femmes infectées (chez lesquelles le parasite circule) et enceintes ne sont pas traitées, par crainte d'une hypothétique toxicité pour le fœtus.

En 1986, à la frontière entre la Thaïlande et la Birmanie, la mortalité maternelle due au paludisme s'élevait à 1000 pour 100000 naissances vivantes (comparée à 8 pour 100000 en France). Dans cette zone de forte chimiorésistance de $P$. falciparum (et plus récemment de $P$. vivax), nous avons pu étudier l'efficacité, la tolérance et la pharmacocinétique des antipaludiques les plus récents [4]. Ces études ont permis, en conjonction avec un dépistage systématique, d'éliminer la mortalité maternelle et de fournir des données uniques sur les médicaments aujourd'hui déployés dans le reste du monde (Figure I). En particulier nous avons montré que les doses administrées (définies à partir d'études menées chez des hommes adultes) étaient inadaptées chez les femmes enceintes pour la plupart des médicaments. Pour des raisons multiples: métaboliques (proguanil), volume de distribution et clairance augmentés pendant la grossesse [atovaquone (Malarone $\left.{ }^{\circledast}\right)$, artésunate, artéméther-luméfantrine (Coartem $\left.{ }^{\oplus}\right)$ ], les niveaux plasmatiques des principes actifs et de leurs métabolites sont insuffisants avec pour conséquence une efficacité diminuée. La disponibilité du Fansidar (sulfadoxine-pyriméthamine) est aussi modifiée comme l'ont indiqué des études récemment menées en Afrique. Donc les recommandations sur l'utilisation de ce médicament en traitement préventif intermittent, indication très fréquente en Afrique, sont sans doute erronées. Les données sur les cinétiques de la quinine et de la pipéraquine manquent et celles sur la méfloquine sont incomplètes. Il est donc encore aujourd'hui très difficile de définir des recommandations pour l'utilisation des antipaludiques chez la femme enceinte, en s'appuyant sur des données cliniques et biologiques fiables [5].

\section{Artémisinine et toxicité fœtale}

Les dérivés de l'artémisinine (artésunate, artéméther) sont maintenant recommandés par l'OMS dans toutes

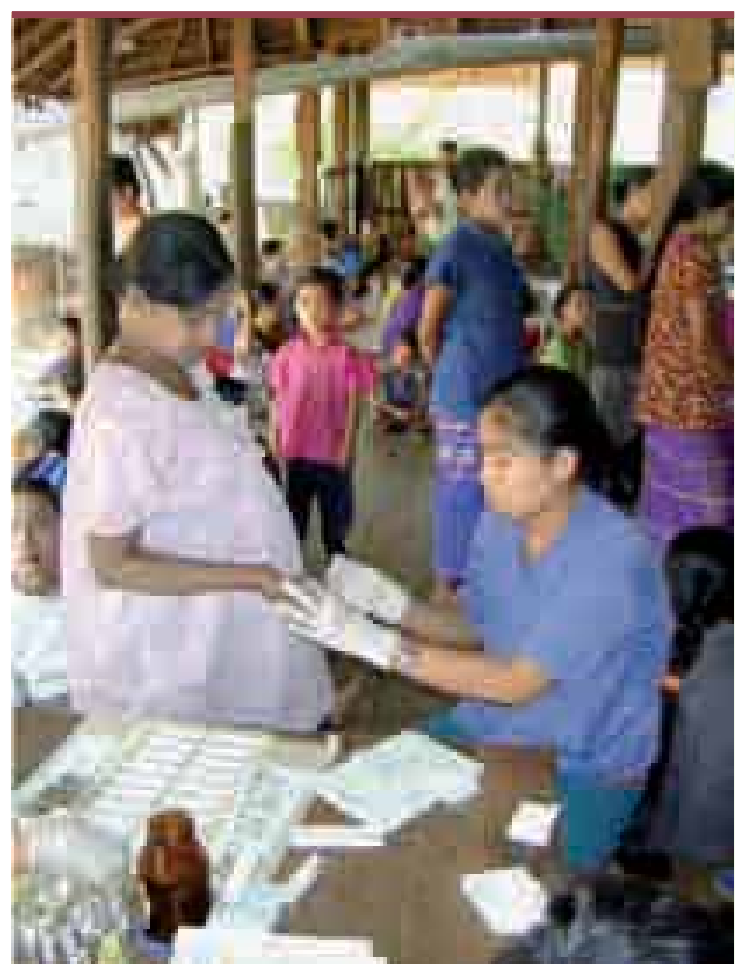

Figure 1. Dépistage hebdomadaire du paludisme dans les consultations prénatales du SMRU. 
les zones d'endémie palustre en association avec une molécule dite partenaire afin de limiter l'apparition de résistances. Leur utilisation pendant la grossesse est délicate à cause de la toxicité embryonnaire constatée chez l'animal. Cette classe de médicaments a une affinité particulière pour les cellules riches en fer et provoquent la destruction des cellules embryonnaires primitives, à des doses supérieures à celles qui sont utilisées chez l'humain. Dans notre expérience chez plus de 1000 femmes enceintes, aucune toxicité pour le fœtus n'a été observée. Néanmoins la période critique de la grossesse se situant dans les premières semaines, il est difficile d'exclure complètement une toxicité fœtale rare, la femme elle-même ne sachant pas toujours qu'elle est enceinte. Dans le traitement du paludisme grave, l'artésunate injectable réduit la mortalité de $35 \%$ par rapport à la quinine [6]. II semble donc difficile de refuser ce traitement à une femme enceinte par crainte d'une hypothétique toxicité fœtale, alors que la mortalité liée au paludisme cérébral dépasse souvent $50 \%$ pendant la grossesse. Enfin, les doses des dérivés de l'artémisinine doivent être adaptées chez les femmes enceintes car les études de pharmacocinétique montrent que les taux plasmatiques du métabolite principal (la dihydro-artémisinine) sont inférieurs de $50 \%$ pendant la grossesse [7].

\section{Conclusion}

La prise en charge médicamenteuse du paludisme de la femme enceinte souffre d'un manque cruel de données cliniques et biologiques, ce qui ne permet pas de guider l'utilisation des antipaludiques indispensables pour le traitement et la prévention de cette infection très répandue et responsable d'une mortalité intolérable. Dans nos sociétés de plus en plus rétives au risque (même minime), cela aboutit à la situation paradoxale actuelle où des femmes atteintes de paludisme reçoivent des médicaments sûrs, mais inefficaces. De nouvelles entités telles que le Consortium paludisme et grossesse ${ }^{1}$ permettront

http://www.mip-consortium.org peut être la réalisation d'études à grande échelle offrant toutes les garanties éthiques et scientifiques qui seront la source d'informations indispensables à l'établissement des politiques nationales de santé. $\diamond$

Malaria in pregnancy: a therapeutic dilemma

\section{CONFLIT D'INTÉRÊTS}

L'auteur déclare n'avoir aucun conflit d'intérêts concernant les données publiées dans cet article.

\section{RÉFÉRENCES}

1. Desai M, Ter Kuile F0, Nosten F, et al. Epidemiology and burden of malaria in pregnancy. Lancet Infect Dis 2007 ; 7 : 93-104.

2. Nosten F, McGready R, Simpson JA, et al. Effects of Plasmodium vivax malaria in pregnancy. Lancet 1999 ; $354: 546-9$.

3. McGready R, Cho T, Hkirijaroen L, et al. Quinine and mefloquine in the treatment of multidrug-resistant Plasmodium falciparum malaria in pregnancy. Ann Trop Med Parasitol 1998; 92 : 643-53.

4. McGready R, Nosten F. The thai-burmese border: drug studies of Plasmodium falciparum in pregnancy. Ann Trop Med Parasitol 1999 ; 93 (suppl 1): S19-23.

5. Ward SA, Sevene $\varepsilon J$, Hastings IM, et al. Antimalarial drugs and pregnancy : safety, pharmacokinetics, and pharmacovigilance. Lancet Infect Dis $2007 ; 7: 136-44$.

6. Dondorp A, Nosten F, Stepniewska K, et al. Artesunate versus quinine for treatment of severe Falciparum malaria : a randomised trial. Lancet $2005 ; 366: 717-25$.

7. McGready R, Stepniewska K, Ward SA, et al. Pharmacokinetics of dihydroartemisinin following oral artesunate treatment of pregnant women with acute uncomplicated Falciparum malaria. Eur J Clin Pharmacol $2006 ; 62: 367-71$.

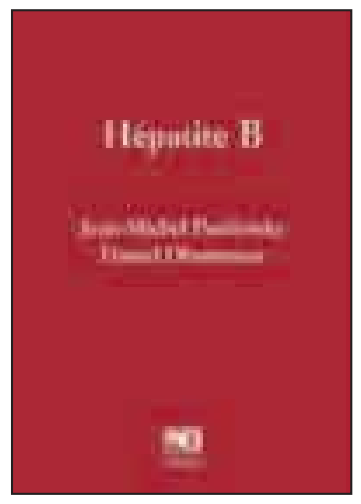

ISBN : 978-2-8425-4131-6 576 pages

\section{Bon de commande}

À retourner à EDK, 2, rue Troyon - 92316 Sèvres Cedex

Tél. : 0155641393 - Fax : 0155641394 - E-mail : edk@edk.fr

NOM : Prénom :

Adresse :.

Code postal : Ville :

Pays :

Fonction :

Je souhaite recevoir l'ouvrage Hépatite B : $60 €+3 €$ de port $=63 €$ TTC

$\square$ exemplaire, soit un total de €...

$\square$ Par chèque, à l'ordre de $\mathbf{E} \mathbf{D} \mathbf{K}$

Par carte bancaire : $\square$ Visa $\square$ Eurocard/Mastercard

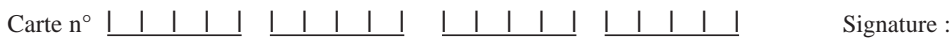

Date d'expiration : $\quad \leq 1|\perp|$

$\mathrm{N}^{\circ}$ de contrôle au dos de la carte : $\quad|\quad| \quad \mid$

\section{TIRÉS À PART}

F. Nosten 